\title{
Characterization of Normal Murine Carpal Bone Development Prompts Re-Evaluation of Pathologic Osteolysis as the Cause of Human Carpal-Tarsal Osteolysis Disorders
}

\author{
Syndia Lazarus, ${ }^{* \dagger \ddagger}$ Hsu-Wen Tseng, ${ }^{\dagger \uparrow}$ Felicity Lawrence, Maria A. Woodruff, Emma L. Duncan, ${ }^{* \dagger \|}$ and Allison R. Pettit*§币
}

\begin{abstract}
From the Translational Research Institute, ${ }^{*}$ Brisbane; the University of Queensland Diamantina Institute, ${ }^{\dagger}$ the Mater Research Institute-UQ, ${ }^{\top}$ and the University of Queensland Centre for Clinical Research, ${ }^{\S}$ Faculty of Medicine, The University of Queensland, Brisbane; the Department of Endocrinology and Diabetes, ${ }^{\ddagger}$ Royal Brisbane and Women’s Hospital, Brisbane; and the Institute of Health and Biomedical Innovation, Queensland University of Technology, Brisbane, Queensland, Australia
\end{abstract}

Accepted for publication

May 4, 2017.

Address correspondence to Allison R. Pettit, Ph.D., Mater Research Institute, University of Queensland, The Translational Research Institute, 37 Kent St., Woolloongabba, Brisbane, QLD 4102, Australia. E-mail: allison. pettit@mater.uq.edu.au.

\begin{abstract}
Multicentric carpal-tarsal osteolysis; multicentric osteolysis, nodulosis, and arthropathy; and Winchester syndromes, skeletal dysplasias characterized by carpal/tarsal and epiphyseal abnormalities, are caused by mutations in v-maf musculoaponeurotic fibrosarcoma oncogene ortholog B (MAFB), matrix metalloproteinase (MMP) 2, and MMP14, respectively; however, the underlying pathophysiology is unclear. Osteoclast-mediated osteolysis has been regarded as the main mechanism, but does not explain the skeletal distribution. We hypothesized that MAFB, MMP-2, and MMP-14 have integral roles in carpal/tarsal and epiphyseal bone development. Normal neonatal mouse forepaws were imaged by micro-computed tomography and examined histologically. Murine forepaw ossification occurred sequentially. Subarticular regions of endochondral ossification showed morphologic and calcification patterns that were distinct from archetypical physeal endochondral ossification. This suggests that two different forms of endochondral ossification occur. The skeletal sites showing the greatest abnormality in the carpal-tarsal osteolysis syndromes are regions of subarticular ossification. Thus, abnormal bone formation in areas of subarticular ossification may explain the site-specific distribution of the carpal-tarsal osteolysis phenotype. MafB, Mmp-2, and Mmp-14 were expressed widely, and tartrate-resistant acid phosphatase staining notably was absent in the subarticular regions of the cartilage anlagen and entheses at a time point most relevant to the human osteolysis syndromes. Thus, abnormal peri-articular skeletal development and modeling, rather than excessive bone resorption, may be the underlying pathophysiology of these skeletal syndromes. (Am J Pathol 2017, 187: 1923-1934; http://dx.doi.org/10.1016/j.ajpath.2017.05.007)
\end{abstract}

Skeletal dysplasias are rare genetic disorders that cause abnormal skeletal development and growth. ${ }^{1}$ Within the osteolysis group of skeletal dysplasias are three syndromes with overlapping features: multicentric carpal-tarsal osteolysis syndrome (Mendelian Inheritance in Man; MIM166300); multicentric osteolysis, nodulosis, and arthropathy (also called Torg syndrome or nodulosis arthropathy osteolysis syndrome; MIM259600); and Winchester syndrome (MIM277950). ${ }^{1}$ These conditions are caused, respectively, by mutations in $\mathrm{v}$-maf musculoaponeurotic fibrosarcoma oncogene ortholog B $(M A F B),{ }^{2}$ matrix metalloproteinase $2(M M P 2),{ }^{3}$ and MMP14. ${ }^{4}$ Loss-of-function mutations cause the latter two conditions, ${ }^{3,4}$ however, the effect of multicentric carpal-tarsal osteolysis syndrome causing $M A F B$ mutations on protein function is not yet clear.

\footnotetext{
Supported by National Health and Medical Research Council Medical/ Dental Postgraduate Research Scholarship GNT1056015 (S.L.), Royal Brisbane and Women's Hospital Ph.D. Scholarship (S.L.), the Department of Endocrinology at the Royal Brisbane and Women's Hospital (S.L.), and the Mater Foundation and the Australian and New Zealand Bone and Mineral Society Gap Fellowship (A.R.P.). The Translational Research Institute Preclinical Imaging Facility receives funding support from Therapeutic Innovation Australia.
}

Disclosures: None declared. 
All three conditions are characterized by site-specific bone abnormalities, with progressive destruction of carpal and tarsal bones and epiphyses of many long bones evident on serial radiographs (Figure 1) and are assumed to be a consequence of excessive osteolysis. Because carpotarsal osteolysis is the overriding common feature of all three syndromes, they are henceforth referred to as carpotarsal osteolysis disorders (CTODs). Contractures across the involved joints are another common feature. ${ }^{2,6-12}$ Affected individuals typically present with swelling of their wrist and/or feet in early childhood, with quiescence during adolescence. Bisphosphonates have been used in trials for both multicentric carpal-tarsal osteolysis syndrome and multicentric osteolysis, nodulosis, and arthropathy, with anecdotal improvement in pain and swelling in some cases but no objective evidence of altered disease progression. ${ }^{11,13,14}$ Similarly, glucocorticoid therapy, nonsteroidal anti-inflammatory drugs, or immunomodulatory therapy has provided symptom relief but not altered bone phenotype or disease progression. ${ }^{15-18}$

Individuals with multicentric osteolysis, nodulosis, and arthropathy have recessive mutations of $M M P 2$ and reduced MMP-2 expression. ${ }^{3,19}$ MMP-2 is a gelatinase with a wide variety of bone-relevant substrates including collagens and proteoglycans. ${ }^{20}$ It is secreted as a pro-enzyme, and then activated by MMP-14. Winchester syndrome has been described in only two sisters, homozygous for a MMP14 mutation, who had severely reduced MMP-14 activity and consequently had decreased active MMP- $2 .{ }^{4}$ In contrast, the molecular consequences of $M A F B$ mutations are unclear. MAFB has key roles in osteoclast and macrophage differentiation, intuitively suggesting that mutations affect osteoclasts and, consequently, osteolysis. However, MAFB is up-regulated during early osteoclast differentiation but down-regulated in late differentiation, ${ }^{21,22}$ suggesting direct influence over myeloid commitment rather than osteoclast formation and function.

Interestingly, both MAFB and MMP-2 are implicated in bone formation. MAFB is expressed by cartilage-producing chondrocytes within the physis (growth plate), and negatively regulates aggrecan expression in vitro. ${ }^{23,24}$ Thus, $M A F B$ mutations causing multicentric carpal-tarsal osteolysis syndrome may affect mesenchymal lineage pathways, including chondrocyte and/or osteoblast function. MMP-2 is present within articular and/or periarticular human fetal and adult chondrocytes, and expression is increased in osteoarthritic cartilage. ${ }^{25,26} \mathrm{Mmp}^{-1-}$ bone marrow has reduced osteoblastic and osteoclastic potential. ${ }^{27}$ Thus, reduced MMP-2 activity in both multicentric osteolysis, nodulosis, and arthropathy and Winchester syndrome might alter bone and/or cartilage formation.

Skeletal development is complex. Bone is formed either through intramembranous ossification (direct bone formation without a cartilage intermediary; eg, the cranial vault) or endochondral ossification (in which bone replaces a cartilage template; eg, limb long bones). The human hand undergoes sequential ossification of its eight carpal bones, beginning with the capitate and hamate (where primary ossification centers (POCs) appear at approximately 2 to 3 months), and ending with the pisiform at approximately 8 to 11 years. Carpal bones achieve maturity before fusion of the radial and ulnar epiphyses. ${ }^{28}$

Thus, evidence that the CTODs are osteolysis syndromes largely is assumed, and needs reconsideration given the following: i) minimal benefits of anti-osteolytic treatments, ii) recent elucidation of their molecular causes, and iii) site specificity of the skeletal phenotype. Thus, we investigated postnatal forepaw development in normal mice to provide insight into potential site-specific developmental mechanisms within bones affected by CTODs.
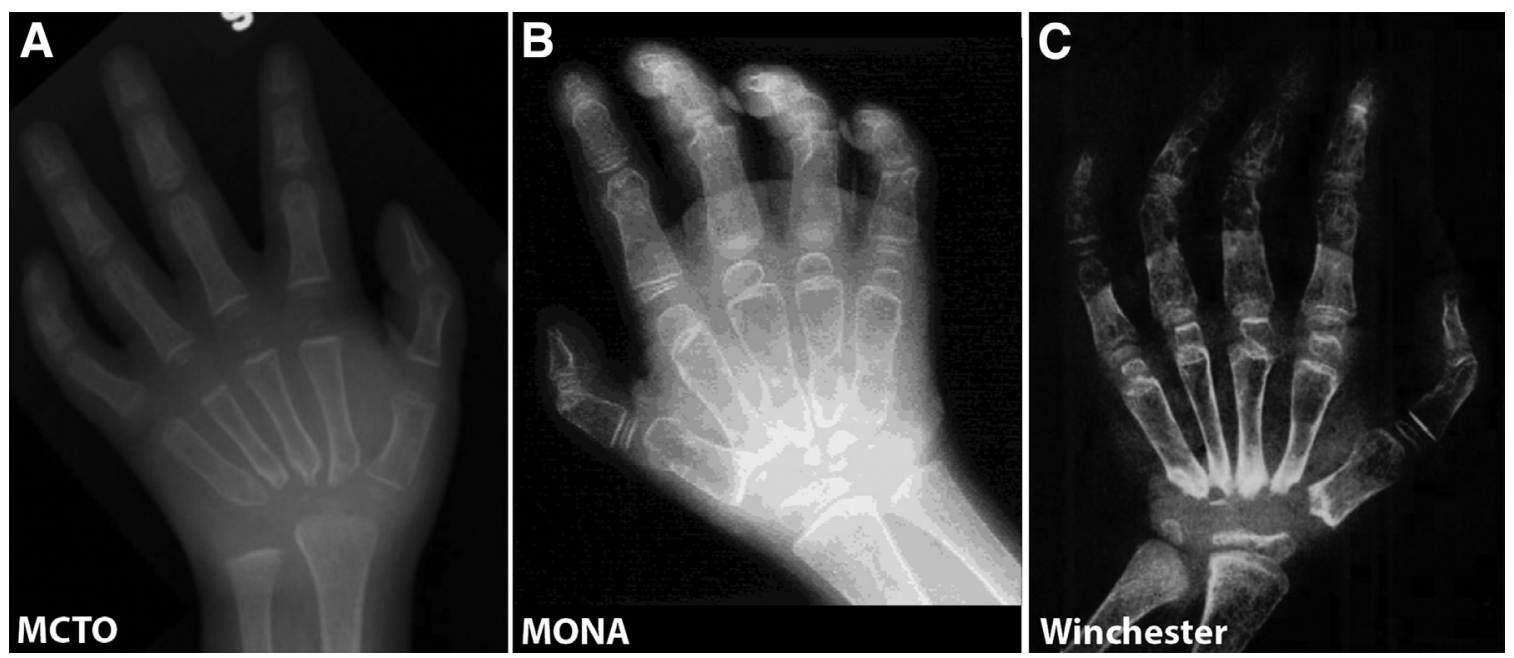

Figure 1 X-rays from children affected by carpal-tarsal osteolysis (MCTO) syndromes. A 3-year-old with (MCT0) syndrome (A), ${ }^{2}$ a 4-year-old with multicentric osteolysis, nodulosis, and arthropathy (MONA) (B), ${ }^{5}$ and a 6 -year-old with Winchester syndrome (C). ${ }^{6}$ Reprinted from Zankl et al, ${ }^{2}$ Al Aqeel et al, ${ }^{5}$ and Winchester et al, ${ }^{6}$ with permission from Elsevier, John Wiley and Sons, and the American Journal of Roentgenology, respectively. 


\section{Materials and Methods}

\section{Research Animals and Tissue Collection}

All mice were treated in accordance with the Australian code of practice for the care and use of animals. Ethics approval was obtained from The University of Queensland Health Sciences Animal Ethics Committee (UQCCR/056/ 12/NHMRC and MATER/052/14/RBWH). Forepaws were collected from wild-type $\mathrm{C} 57 \mathrm{Bl} / 6$ mice on postnatal days 2 , $4,6,8$, and 12 , and at week $8(n=4 /$ age $)$. Overlying soft tissue was removed followed by fixation using $4 \%$ paraformaldehyde. Left paws were imaged by micro-computed tomography (micro-CT) followed by resin embedding as previously described. ${ }^{29}$ Right paws were decalcified in $14 \%$ EDTA and processed for paraffin embedding as previously described. ${ }^{30}$

\section{Micro-CT}

Left forepaws were imaged using a multimodality Platform Inveon preclinical micro-PET/CT scanner (Siemens, Berlin, Germany) unfiltered at $60 \mathrm{kV}$ and $500 \mu \mathrm{A}$. Gantry total rotation was $360^{\circ}$ with 512 rotation steps using an exposure time of $430 \mathrm{~ms} /$ frame. The effective pixel size was $34 \mu \mathrm{m}$. Image reconstruction was performed with a down sample factor of one using Cobra Reconstruction software version 6.3.39 (Exxim Computing Corporation, Pleasanton, CA). Three-dimensional reconstruction of the bone was analyzed with Inveon Research Workplace software version 1.5 (Siemens). An atlas of micro-CT images of the adult mouse skeleton was used to identify developing bones. ${ }^{31}$

\section{Histology and Immunohistochemistry}

Sections were cut in the coronal plane, enabling maximum simultaneous visualization of independent carpal bones, with triangular, navicular-lunate, lesser multangular, capitate, and hamate sampled routinely. Serial sections $(5 \mu \mathrm{m})$ were cut from paraffin-embedded tissues and deparaffinized with xylene and graded ethanol. These were stained either using standard histologic protocols or immunohistochemistry. Hematoxylin and eosin was used to assess morphologic features, $0.1 \%$ toluidine blue staining was used to identify proteoglycan-rich tissues including cartilage, and tartrate-resistant acid phosphatase (TRAP) activity was used to identify osteoclasts. ${ }^{32}$ Immunohistochemistry was performed as previously described, ${ }^{30}$ using the following primary antibodies: anti-MafB (rabbit anti-mouse; Novus Biologicals, Littleton, CO), anti-Mmp2 (rabbit anti-human; Abcam, Cambridge, UK), anti-Mmp14 (rabbit anti-human; Abcam), anti-F4/80 (rat anti-mouse; Abcam), and antiosteocalcin (rabbit anti-mouse; Enzo Life Sciences, Farmingdale, NY) antibodies. Staining of serial sections with matched isotype control antibodies (normal rabbit $\mathrm{IgG}$; Abcam; or rat IgG2b; Biolegend, San Diego, CA) was used to confirm the specificity of staining. Antigen retrieval was required for anti-MafB and anti-Mmp14 (0.37\% trypsin for 8 minutes), anti-Mmp2 and anti-osteocalcin (proteinase $\mathrm{K}$ for 5 minutes), and anti-F4/80 (0.37\% trypsin for 10 minutes or heat method in sodium citrate buffer). Primary antibodies were incubated overnight at $4^{\circ} \mathrm{C}$, except for anti-F4/80, which was incubated for 2 hours at room temperature. Sections were counterstained with Mayer's hematoxylin, mounted, and viewed using an Olympus BX50 microscope (Olympus, Tokyo, Japan) or scanned using a Leica SCN400 slide scanner (Leica Microsystems, Wetzlar, Germany), and viewed using Digital Image Hub for Leica version 4.0.5 (SlidePath, Dublin, Ireland).

\section{Results}

\section{Murine Forepaw Ossification Is Sequential, with Distinct Patterns of Calcification}

The murine carpus consists of 10 bones arranged in two parallel rows. ${ }^{31}$ Forepaw micro-CT imaging showed complete radiolucency in the carpus region on day 2 , and the distal ulna and radius also were radiolucent (Figure 2A). Thus, these bones had not commenced ossification. On day 4, tiny foci of radiodensity indicating calcification were detectable in the pisiform, triangular, and hamate bones (Figure 2B). By day 6, these radiodensities had increased in size; small centers of radiodensity also were visible in the capitate, lesser multangular and navicular-lunate, and in the ulnar styloid process (Figure 2, C and K). On day 8, all 10 carpals showed radiodensity (Figure 2D), and by day 12 the carpals had a similar radiologic appearance to that of the week 8 sexually mature mouse (Figure 2, E and F). The radial and ulnar physes were much narrower but still distinguishable at week 8 (murine physes never fully close). Thus, carpal ossification occurs sequentially in both mice (Figure 2, A-F) and humans (Figure 2, G-J), with formation and mineralization complete by sexual maturity in both species. Individuals affected by CTODs typically present before 3 years of age, a stage of carpal ossification best represented by postnatal day 6 mice (Figure 2, $\mathrm{C}$ and $\mathrm{H}$ ).

Within the carpals, von Kossa staining of sections from micro-CT-imaged forepaws showed that the radiodensity detected on days 6 and 8 was associated with calcified cartilage matrix surrounding hypertrophic chondrocytes of emerging POCs (Figure 2, K-N). Calcium deposition first occurred in the center of each carpal cartilage anlagen, and extended radially. There was sparing of articular cartilage, with a well-defined peripheral zone of uncalcified cartilage evident in the carpals by day 8 (Figure 2, M and N). Calcified cartilage was replaced with calcified bone by week 8 .

Within the distal ulna and radius, the distribution of calcium within the articular pole of the ulnar (Figure 2L) and radial secondary ossification centers (SOCs) (Figure 2, $\mathrm{M}$ and $\mathrm{O}$ ) was similar to that seen in carpal POCs, with extensive calcification of subarticular cartilage occurring by 

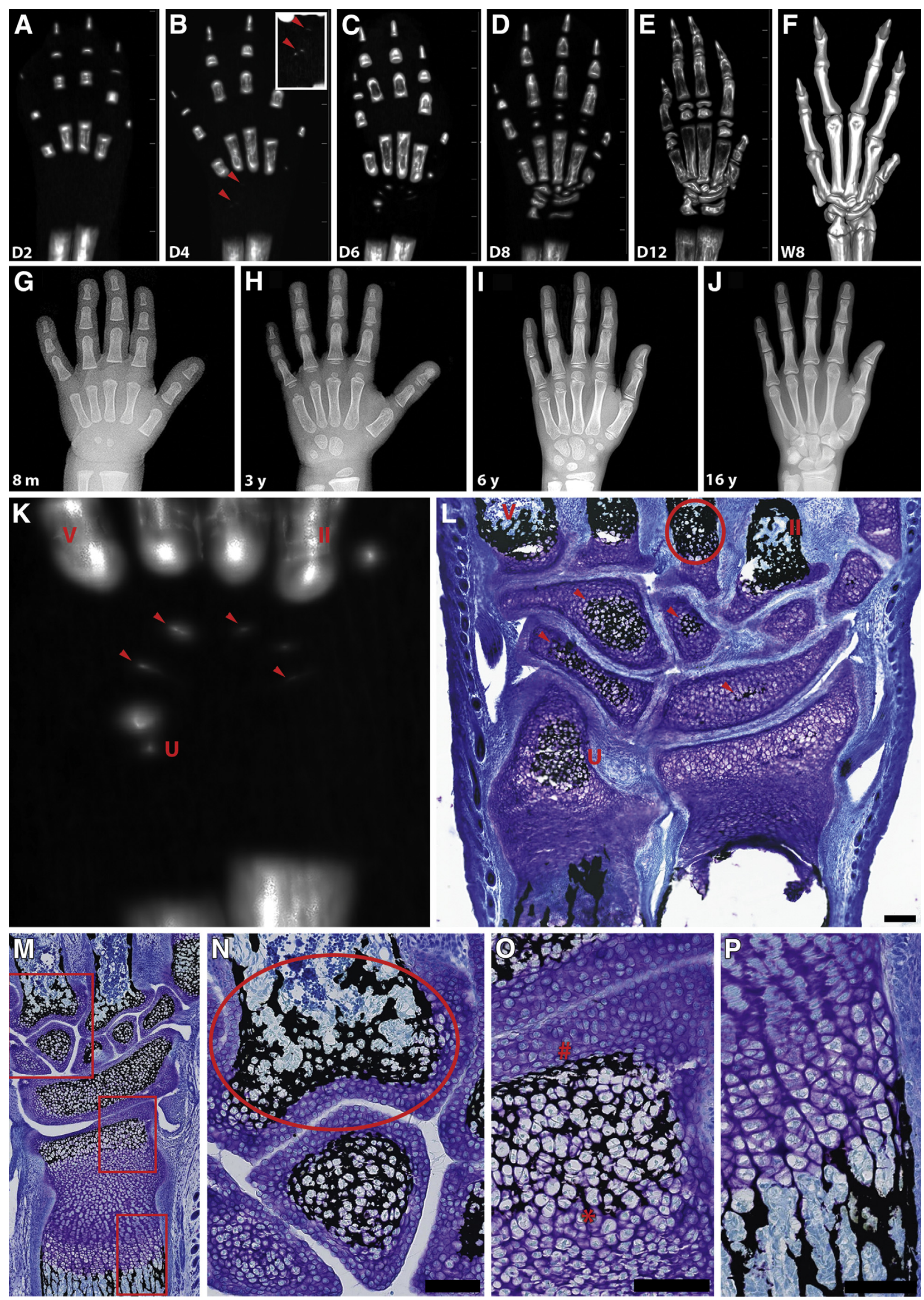
day 8 , and well-defined sparing of articular cartilage. In contrast, the nonarticular end of the SOC had uncalcified hypertrophic cartilage contiguous with proliferative chondrocytes of the physis (Figure 2, M and O).

Within the proximal metacarpals, the D6 proximal second to fifth metacarpals showed uncalcified articular cartilage merging with a thick zone of calcified hypertrophic cartilage (Figure 2L), which, in turn, was contiguous with the marrow cavity. By day 8 , the zone of calcified cartilage in the proximal metacarpals had thinned (Figure 2, M and N). The appearance of the proximal metacarpals resembled that of the carpal POC and subarticular SOC cartilage.

Overall, several patterns of cartilage calcification were evident within the distal murine forelimb during postnatal skeletal maturation, such as: i) subarticular (in which all hypertrophic cartilage matrix was calcified), seen in carpal POCs, and in subarticular cartilage of the proximal metacarpals, distal ulna, and radius; ii) prephyseal (in which there are moderate amounts of both calcified and uncalcified hypertrophic matrix), seen in the distal ulna and radius; and iii) postphyseal (in which very little hypertrophic matrix is calcified) (Figure 2P), seen in the distal ulna and radius. The articular cartilage remained uncalcified at all time points.

\section{Forelimb Bones Developing via Subarticular Ossification Share Morphologic Features that Are Distinct from Physeal Ossification}

Contralateral limb forepaw skeletal tissues were examined using standard histologic approaches (hematoxylin and eosin, not shown, and toluidine blue) (Figure 3). Carpals were composed entirely of avascular proteoglycan-rich cartilage on day 2 (Figure 3A). Chondrocytes appeared to be distributed isotropically on day 2 (Figure 3, A and G), lacking the highly organized columnar appearance of typical physes in the sectional plane used. On day 4 (Figure 3B) and day 6 (Figure 3, C and H), the central chondrocytes had larger lacunae and were hypertrophic.

Cartilage canals first were observed on day 6 (Figure 3, C and $\mathrm{H}$ ) and appeared sequentially, following the same temporal and spatial sequence as for the calcification described earlier. Extensive excavation of the central cartilage matrix and early bone formation was evident by day 10 in all carpals (not shown); day 12 samples had a similar appearance (Figure 3, E and K). By week 8, all carpals were composed predominantly of mature bone, with a peripheral rim of mature articular cartilage (Figure 3, F and L).

At all ages examined, variation in intensity in toluidine blue staining differentiated the carpals into peripheral and central zones (Figure 3, G, H, K, and L); the former marked the future articular cartilage and the latter delineated the region for ossification (Figure 3, G, H, K, and L). Before excavation of the central zone (relevant for all carpals on days 2 to 4 , some carpals on days 6 to 8 ), the peripheral zone was defined by less-intense toluidine blue staining compared with the cartilage matrix of the central zone (Figure 3G), suggesting differential proteoglycan content. After excavation of the central zone (seen from day 6 onward), the peripheral zone persisted as cartilage and therefore continued to stain purple, whereas the central zone was replaced progressively with low-toluidine-blue-stained bone (Figure 3, K and L). Chondrocytes within the peripheral zone were small and round at all ages, whereas chondrocytes within the central zone became progressively more hypertrophic before vascular invasion of the matrix occurred.

The subarticular poles of the distal ulnar and radial SOCs were similar morphologically to carpal POCs at each time point (Figure 3, A-E). Proliferating chondrocytes at the opposite pole (ie, prephyseal chondrocytes) had a different organizational structure. This was apparent on day 6 (Figure 3C) and day 8 (Figure 3D), but was appreciated most readily on day 12 (Figure 3E), where proliferating chondrocytes adjoined a short zone of hypertrophic chondrocytes (Figure 3E); there were fewer proliferating chondrocytes and almost no hypertrophic chondrocytes visible in the subarticular pole (Figure 3E).

The ossifying second to fifth metacarpals had a typical physis at the distal (physeal) ends (Figure 3, I and J). The proximal (nonphyseal) ends had hypertrophic cartilage fusing directly with the POC, without the columnar organization of a physis (Figure 3, I and J). Cartilage of the nonphyseal end rapidly decreased in depth during early postnatal life (Figure 3, A-E, I, and J).

The cartilage of the subarticular ossification centers (seen in the carpals, distal ulna and radius, and second to fifth proximal metacarpals) (Figure 3, A-D) was similar morphologically and had an isotropic arrangement

Figure 2 Micro-computed tomography (micro-CT) and histologic examination of normal murine carpal development shows sequential ossification. A-F: Images of micro-CT scans of murine forepaws from postnatal days 2 (A), 4 (B), 6 (C), 8 (D), 12 (E), and week 8 (F) show sequential ossification of the wrist. Increased contrast settings permit appreciation of small foci of radiodensity in the carpal region on day 4 of postnatal development (inset). G-J: Sequential ossification also is seen in the human wrist (reprinted from Gilsanz and Ratib ${ }^{33}$ with permission from Springer). H: Murine carpal ossification on day 6 corresponds best to human carpal ossification at age 3 years. $\mathbf{K}$ and $\mathbf{L}$ : The appearance of day 6 ossification centers on micro-CT (K) parallel areas of calcified cartilage on histologic examination with von Kossa/McNeal's Tetrachrome staining of the same specimen (L), calcified hypertrophic cartilage of the proximal metacarpal is indicated with a circle. Arrowheads show corresponding ossification centers across micro-CT and histologic specimens [not all primary ossification centers (POCs) are visible in this histologic plane of sectioning; the pisiform POC is the largest ossification center present, but lies anterior (palmar) to the plane of sectioning]. M: Day 8 forepaw. Red boxes are shown at higher magnification in N-P. N: Day 8 proximal third metacarpal and capitate (hypertrophic cartilage of the proximal metacarpal is indicated by an oval). $\mathbf{0}$ : Radial prephyseal chondrocytes of the distal epiphysis, with different patterns of calcification at the articular (hash mark) compared with the nonarticular end (asterisk). P: Postphyseal chondrocytes of the metaphysis. Scale bars: $100 \mu \mathrm{m}$ $(\mathbf{L}, \mathbf{N}-\mathbf{P})$. Original magnification: $\times 20(\mathbf{L}$ and $\mathbf{N}-\mathbf{P}) ; \times 4(\mathbf{M})$. D, day; II, second metacarpal; $\mathrm{m}$, month; $\mathrm{U}$, secondary ossification center of the distal ulna; $\mathrm{V}$, fifth metacarpal; W, week; $y$, year. 
compared with the ulnar and radial physes. This distribution mirrors the findings of the mineralization studies described earlier, which is further suggestive evidence that these regions undergo a similar ossification process, whereas the ulnar and radial physes showed the welldescribed columnar organization of traditional long-bone growth plates.

\section{MafB, Mmp-2, and Mmp-14 Are Expressed in Peripheral Carpal Chondrocytes}

The distribution of the proteins mutated in CTODs (MafB, Mmp-2, and Mmp-14) was determined using immunohistochemistry. Because MafB has an established role in macrophage and osteoclast differentiation, staining for the macrophage-restricted F4/80 antigen and TRAP activity (osteoclasts) also was performed.

In the carpal peripheral zone, chondrocytes almost universally were $\mathrm{MafB}^{+}$(Figure $4, \mathrm{~A}, \mathrm{D}, \mathrm{J}$, and $\mathrm{M}$ ) across the 8-week postnatal skeletal developmental period. Staining predominantly was nuclear, consistent with its role as a transcription factor, but cytoplasmic staining also was seen (Figure 4D and Supplemental Figure S1, A and B). Mmp-2 staining within the carpal peripheral zone also showed consistent staining of most, if not all, chondrocytes, with a cytoplasmic and nuclear protein distribution (Figure 4, B, E, $\mathrm{K}$, and $\mathrm{N}$ ). In addition, there was patchy Mmp-2 cartilage matrix staining, most noticeable from day 4 onward (Figure 4, B, E, K, and N), sometimes involving the articular surface. This could be distinguished readily from lowlevel nonspecific matrix staining seen in isotype controls (Figure 4, P-S, and Supplemental Figure S1, C and D). Peripheral zone chondrocytes also consistently were Mmp$14^{+}$(Figure 4, C, F, L, and O). Mmp-14 staining distribution also was consistent with its known distribution as a cell surface protein. Peripheral cartilage remained negative for both F4/80 expression and TRAP activity at all time points (not shown).

\section{MafB, Mmp-2, and Mmp-14 Are Expressed Highly during Carpal Excavation and in Newly Formed Bone}

On day 2, most central zone chondrocytes were $\mathrm{MafB}^{+}$, Mmp- $2^{+}$, and $\mathrm{Mmp}-14^{+}$(Figure 4, A-C). However, staining intensity was more variable than in smaller peripheral zone chondrocytes; and, occasionally, central zone chondrocytes lacked specific staining, particularly for MafB (Figure 4A) and Mmp-14 (Figure 4C). Similar observations were made on day 4 . By day 6 , when chondrocyte hypertrophy clearly had progressed, a larger proportion of central zone chondrocytes were negative for MafB, Mmp-2, and Mmp-14 (Figure 4, D-F, respectively); similar features were observed on day 8. Intense MafB, Mmp-2, and Mmp14 staining was present within the region of initial cartilage matrix breakdown in the few sections that captured these early stages of carpal excavation (Figure 4, G-I), with clear but patchy Mmp-2 staining in the actual matrix surrounding these excavation zones. Many of the $\mathrm{MafB}^{+}, \mathrm{Mmp}^{-}{ }^{+}$, and Mmp- $14^{+}$cells in this region did not resemble chondrocytes morphologically, some had an elongated and/or ramified appearance reminiscent of activated macrophages (Figure $4 \mathrm{G}$ ). This focal excavation region was no longer present in near-serial sections stained for F4/80 and TRAP activity; consequently, macrophage identity could not be confirmed. However, a section showing radial SOC formation on day 8 contained a region with cells of similar morphology, and these were $\mathrm{F} 4 / 80^{+}$, supporting the presence of macrophages (Figure 5G) (Distributions of MafB, Mmp-2, and Mmp-14 within Other Subarticular Tissues Replicate the Distribution in Carpal Tissues).

Morphologically identifiable bone was present in carpal central zones from day 8 onward; day 12 images (Figure 4, $\mathrm{J}-\mathrm{L}$ ) are provided as representative of this stage in development. Most cells in the vicinity of newly laid/actively forming bone were intensely $\mathrm{MafB}^{+}, \mathrm{Mmp}^{+}{ }^{+}$, and Mmp$14^{+}$(Figure 4, J-L). Although we were unable to ascertain all of the specific positive cell types definitively, at least some of the $\mathrm{MafB}^{+}, \mathrm{Mmp}-2^{+}$, and Mmp- $14^{+}$staining was in osteocalcin ${ }^{+}$osteoblasts (not shown). $\mathrm{MafB}^{+}$osteoclasts were observed occasionally (Figure $4 \mathrm{~J}$ ). Occasional TRAP ${ }^{+}$ osteoclasts were present in the hamate on day 8 , the site of most advanced carpal excavation (Figure 5A); all carpal central zones were $\mathrm{TRAP}^{+}$by day 12 (Figure $5 \mathrm{~B}$ ).

\section{Distributions of MafB, Mmp-2, and Mmp-14 within Other Subarticular Tissues Replicate the Distribution in Carpal Tissues}

The ulnar and radial SOCs showed a similar developmental pattern of MafB, Mmp-2, and Mmp-14 staining to carpal POCs, particularly at the articular pole. Peripheral chondrocytes at the articular pole largely were $\mathrm{MafB}^{+}$ (Figure 5C), Mmp- $2^{+}$, or Mmp- $14^{+}$, but did not express TRAP or F4/80 (not shown). Small prephyseal chondrocytes showed variable MafB (Figure 5D), Mmp-2, and Mmp-14 staining (not shown). Central zone hypertrophic chondrocytes also showed similar staining to carpal POCs at comparable developmental stages, with variable expression of MafB (Figure 5C), Mmp-2, and Mmp-14. As was seen within carpal POCs, the proportion of positive cells in mice on days 6 and 8 (at which time vascular invasion had occurred) decreased compared with younger mice (not shown). On day 8 , one section showed $\mathrm{F} 4 / 80^{+}$macrophages forming a loose line that connected the edge of the radius to the region of cartilage excavation; the line terminated near the developing radial SOC, which contained scattered F4/ $80^{+}$macrophages (Figure 5G). A near-serial section also showed macrophage-like $\mathrm{MafB}^{+}$cells within the radial SOC (Figure 5H).

Within radial and ulnar physes, MafB staining was most intense within the hypertrophic zone (Figure 5E), which is consistent with previously published data. ${ }^{23}$ A similar 

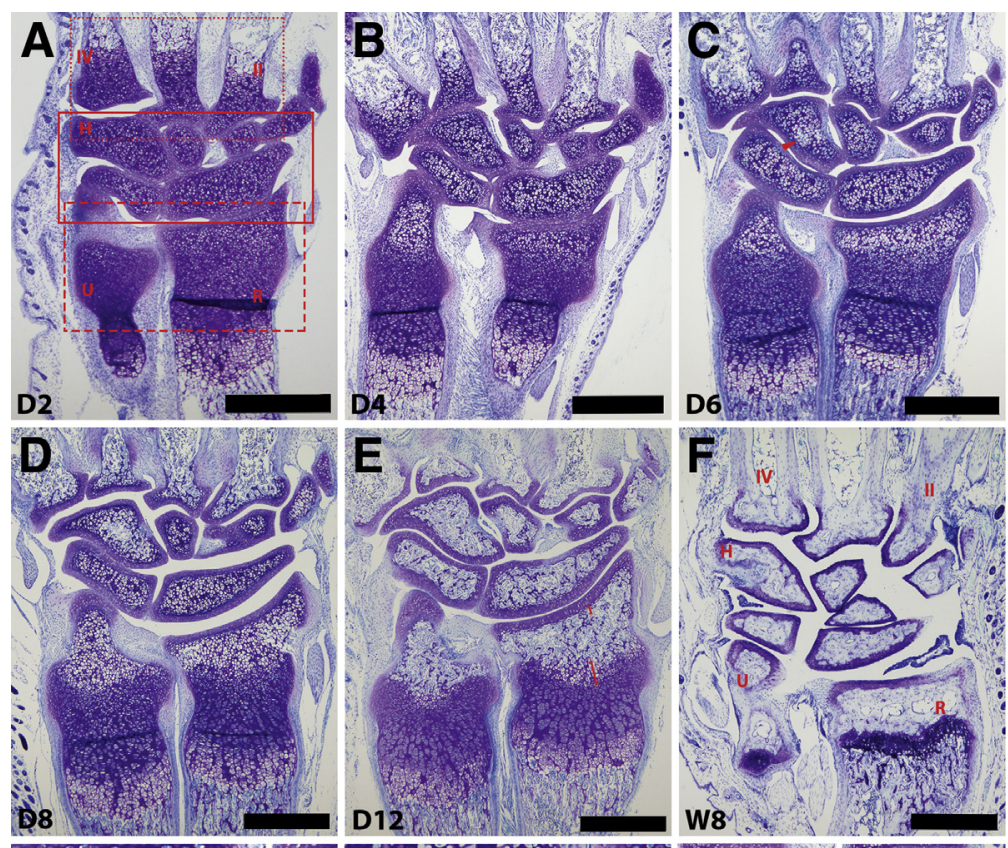

Figure 3 Subarticular regions of ossification are similar morphologically. Toluidine blue staining of murine forepaws on days 2 (A) (solid box indicates carpals; dashed box, distal ulna and radius; dotted box, proximal metacarpals), 4 (B), 6 (C) (arrowhead indicates site of vascular invasion), 8 (D), 12 (E), and week 8 (F) showing progressive maturation of the ossification centers within the wrist based on proteoglycan-rich-cartilage matrix distribution and chondrocyte morphology. Red lines in $\mathbf{E}$ indicate hypertrophic chondrocyte zones in the secondary ossification center (long line) and subarticular pole region (short line). The hamate on postnatal days $2(\mathbf{G}), 6$ (H; arrowhead shows the same site of vascular invasion indicated in $\mathbf{C}), 12$ (K; arrowheads show cartilage remnants in bone), and week 8 (L). The metacarpal on days 4 (I) and $8(\mathrm{~J})$; the proximal end is oriented at the bottom of the page for both $\mathbf{I}$ and $\mathbf{J}$ with asterisks indicating the distal typical physis and hash marks indicating the proximal nonphyseal end. Scale bars: $500 \mu \mathrm{m}(\mathbf{A}-\mathbf{F}, \mathbf{I}$, and $\mathbf{J}) ; 100 \mu \mathrm{m}(\mathbf{G}, \mathbf{H}, \mathbf{K}$, and $\mathbf{L})$. Original magnification: $\times 4(\mathbf{A}-\mathbf{F}, \mathbf{I}$, and $\mathbf{J}) ; \times 20(\mathbf{G}, \mathbf{H}, \mathbf{K}$, and $\mathbf{L})$. C, central zone; D, day; $\mathrm{H}$, hamate; II, second metacarpal; IV, fifth metacarpal; $P$, peripheral zone; $R$, distal radius; $U$, distal ulna; W, week.
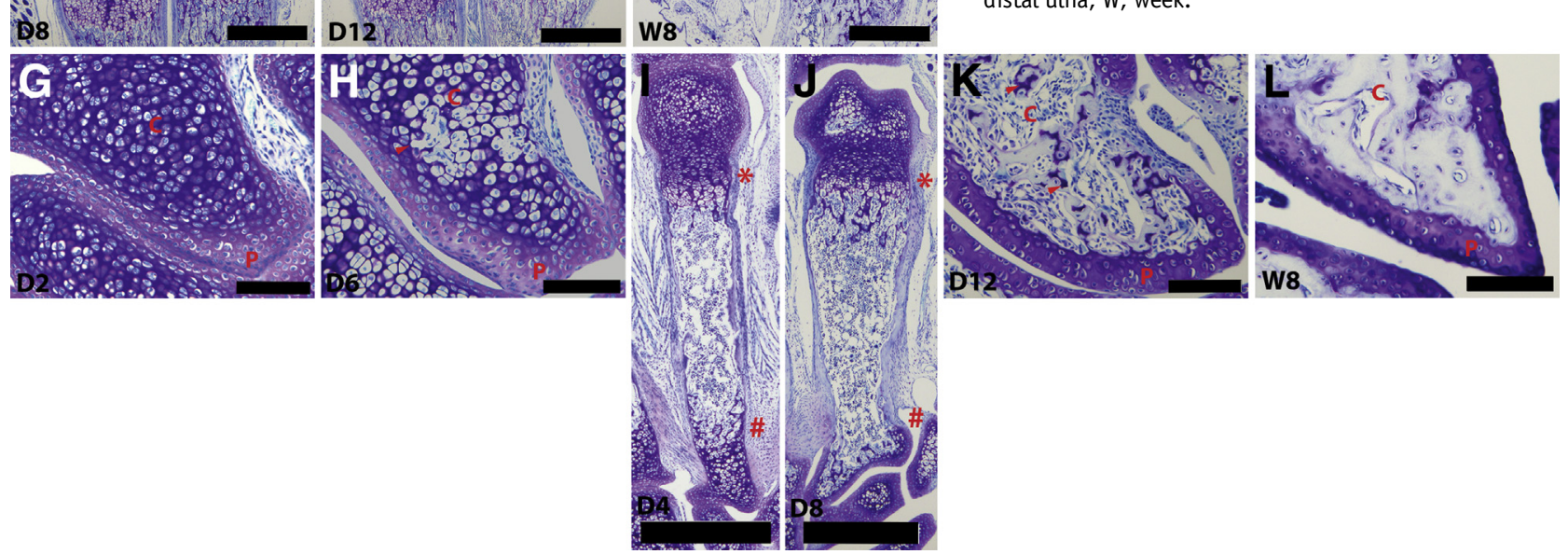

distribution was seen for Mmp-14 staining (not shown); however, all physeal chondrocytes strongly were Mmp- $2^{+}$ (not shown). As expected, the ossification front and new bone within the radial and ulnar POCs contained numerous $\mathrm{TRAP}^{+}$cells, and the matrix also strongly was TRAP ${ }^{+}$(not shown).

Chondrocytes of the proximal second to fifth metacarpals also showed similar MafB (Figure 5F), Mmp-2, and Mmp-14 (not shown) staining to that of the carpal POC.

\section{Entheseal Distribution of Mmp-2 Overlaps but Is Distinct from MafB and Mmp-14}

From day 4 onward, the most intense Mmp-2 staining was concentrated at the entheses (Figure 5, I and J). Isolated, intensely $\mathrm{MafB}^{+}$cells were present at some carpal entheses (not shown), but these areas did not correspond consistently to $\mathrm{Mmp}-2^{+}$entheseal regions. Distal ulnar and radial entheses were Mmp-2 ${ }^{+}$(Figure 5, I and $\mathrm{J}$ ) and $\mathrm{MafB}^{+}$ (Figure 5K). Entheses also weakly were $\mathrm{Mmp}-14^{+}$(not shown). The entheseal matrix adjacent to the metacarpals is markedly Mmp- $2^{+}$in day 4 and older mice (Figure 5I), but only weakly Mmp- $14^{+}$and show minimal MafB expression (not shown). The entheses and adjacent cartilage were negative for TRAP staining (not shown).

\section{Discussion}

The three prototypical CTODs were characterized as osteolytic syndromes based on radiographic changes in the affected bones. ${ }^{6,34,35}$ We have been unable to identify any direct evidence to support osteolysis as the underlying pathophysiology. In fact, the site-specific distribution of affected bones is inconsistent with a genetically driven, generalized osteolytic dysfunction. Very little is known about the mechanisms involved in the formation of normal wrist bones. Therefore, we sought to provide a framework for understanding the site specificity of CTODs by characterizing this developmental process in healthy mice. Our study showed differences in subarticular compared with physeal ossification in wrist bones, both in timing and in cellular organizational structure. We show for the first time that the protein products of the genes mutated in these 


\section{MafB}

Mmp2

\section{Mmp14}

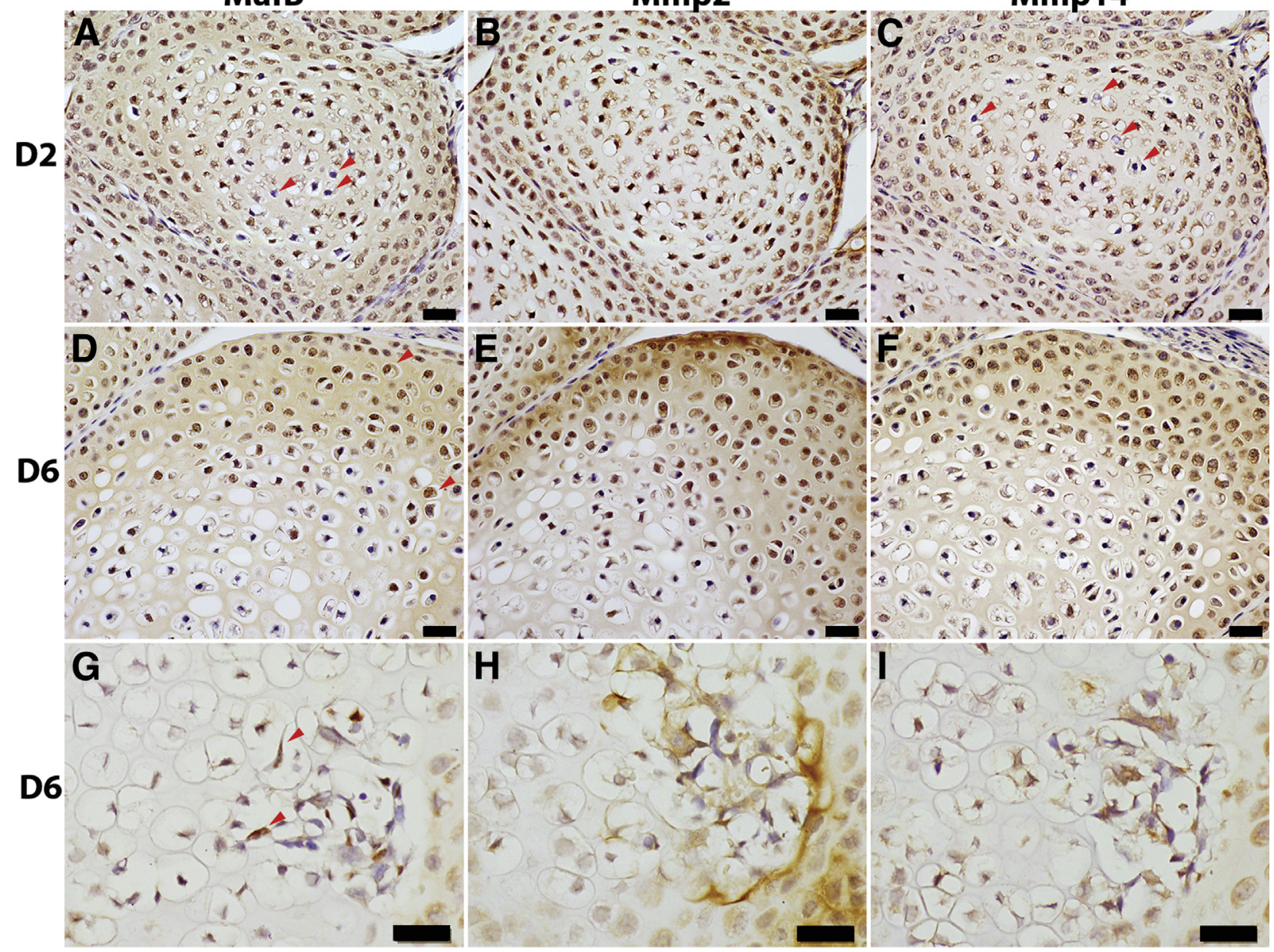

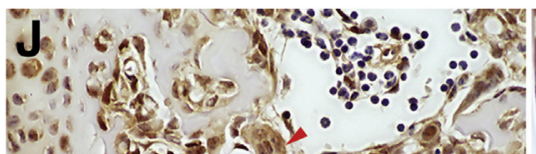

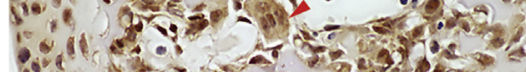

D12
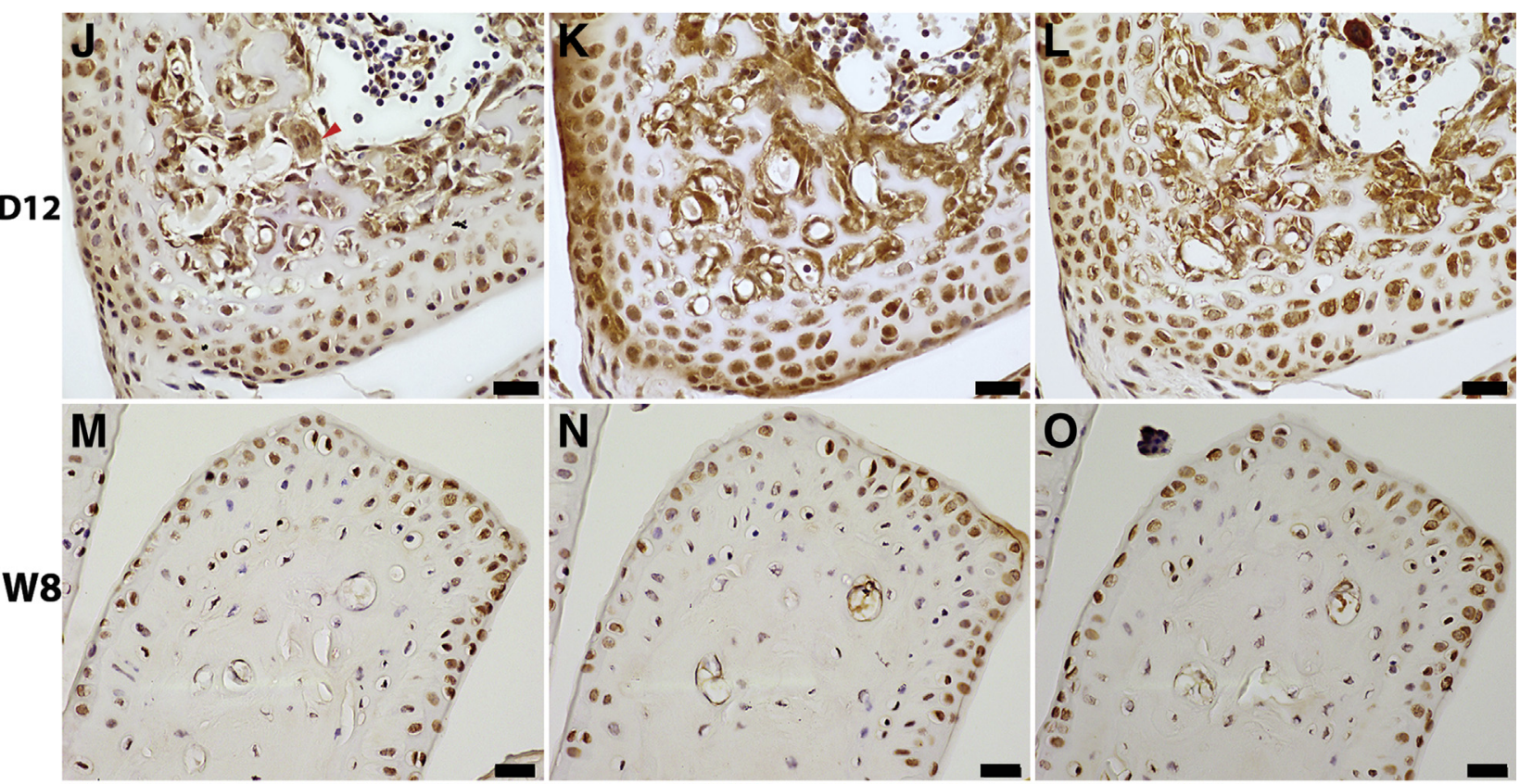

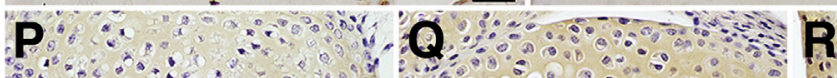

$\mathbf{R}+\mathbf{S}$

Iso 
syndromes were expressed in the affected bones at developmental stages equivalent to the age at which bone abnormalities first manifest. In addition, the timing and distribution of MafB, Mmp-2, and Mmp-14 expression in the relevant wrist bones differed from that observed at sites of classic physeal endochondral ossification, sites that show minimal pathology in CTODs. These observations suggest that distinct osteogenic mechanisms are involved in wrist bone formation, providing unprecedented insight into the observed site-specific distribution of affected bones in CTODs.

We have shown that subarticular and physeal regions undergo distinct variations of endochondral ossification. Specifically, regions of subarticular ossification (ie, carpal POCs, distal radial and ulnar SOCs, and the nonphyseal end of metacarpals) had chondrocytes arranged isotropically and underwent extensive cartilage matrix mineralization before widespread excavation and replacement with bone in the early postnatal period. This contrasts with ossification of the postphyseal regions, where continual proliferation and maturation of chondrocytes occurred within highly organized columns until skeletal maturity (at age 3 months). ${ }^{36}$ Thus, wrist bones affected characteristically in CTODs form by a common endochondral osteogenesis mechanism that varied from the traditional growth plate.

Murine carpal ossification occurred sequentially, in a pattern similar to that of humans. ${ }^{28}$ The polar ossification pattern of murine metacarpals also is seen in humans. ${ }^{37,38}$ Although interspecies differences clearly exist (eg, murine cartilage canals appear only at the time of ossification but appear much earlier in larger mammals ${ }^{39,40}$ ), small animal models still can provide useful information ${ }^{36,41}$ and our data support that wrist bone development is no exception. CTODs usually present before age 3 years, ${ }^{2,6,7,9-11,18}$ an age best represented by murine carpal ossification on day 6 . Importantly, on day 6 , the bones commonly affected in these syndromes predominantly are cartilaginous. Therefore, in contrast to previous assumptions, ${ }^{6,24,35}$ CTODs may share a common pathology of dysfunctional cartilage anlagen development, cartilage mineralization, and/or vascular invasion, all of which are prominent features involved in the bone formation process at the appropriate developmental stages.

Of particular relevance to the osteolytic classification of CTODs, osteoclasts were observed in carpal tissue only after excavation of the extensively calcified cartilage anlagen had commenced. Therefore, osteoclasts were not involved predominantly in the carpal endochondral ossification mechanisms until after radiographic mineralization of the entire cartilaginous bone template was achieved. Abnormal ossification center formation in CTODs is evident before achieving full mineralization. Consequently, the appearance of osteoclasts in the relevant bones does not match the timing of abnormality onset, suggesting that osteoclasts may not be key participants in carpal development at the time of CTOD symptom onset. This raises the possibility that dysfunctional bone formation, rather than osteolysis, may be the underlying mechanism in CTODs.

The similar temporal and anatomic expression patterns of MafB, Mmp-2, and Mmp-14 within the postnatal murine wrist suggest that all three proteins are involved in articular cartilage formation, growth of the underlying cartilage anlagen in preparation for ossification, and/or initiation and establishment of vascular invasion with attendant influx of mesenchymal cells. We observed differences in expression within chondrocytes at subarticular compared with postphyseal sites, and therefore these proteins may mediate or at least reflect some of the observed differences in ossification mechanisms. MafB, Mmp-2, and Mmp-14 were expressed widely in areas of new bone formation, but there was no evidence of differential site specificity of expression at bone formation sites in any of the tissues examined.

Interestingly, arthritic changes characterized by thin and irregular hyaline cartilage and narrow joint spaces have been reported in CTODs. ${ }^{8,42,43}$ Affected individuals also have characteristic facial features that may be a consequence of altered hyaline cartilage modeling. We showed expression of wild-type MafB, Mmp-14, and Mmp-2 within hyaline cartilage of wrist joints. Given that these proteins are the products of genes mutated in these syndromes, it is possible that the causative mutations in CTODs directly impact hyaline cartilage quality. This observation provides a potential direct causal relationship between the genetic mutations and both the arthritic pathology and the CTOS-associated facial phenotypes. Supporting this interpretation, Mmp-14- or Mmp2-deficient mice develop craniofacial abnormalities that reproduce the corresponding human phenotypes. ${ }^{27,44,45}$ Mmp-14- or Mmp-2-deficient mice also have arthritic pathology, with both mouse strains showing articular cartilage destruction. ${ }^{27,44}$

Compellingly, Mmp-14-deficient mice also develop severe osteopenia and wrist kinking, ${ }^{44}$ reproducing CTOS clinical features. ${ }^{4}$ To our knowledge, there is no published histology of the wrist bones of these mice; therefore, clarification of whether the developmental defect mimics the abnormal wrist bone development in CTODs has to be

\footnotetext{
Figure 4 Distributions of MafB, Mmp-2, and Mmp-14 within the carpals are similar. A-0: Immunohistochemical staining of murine carpal tissue for MafB, Mmp-2, and Mmp-14 at different ages: days $2(\mathbf{A}-\mathbf{C}), 6(\mathbf{D}-\mathbf{I}), 12(\mathbf{J}-\mathbf{L})$, and week 8 (M-0). A and C: Arrowheads indicate central chondrocytes that are not expressing MafB or Mmp-14, respectively. G-I: Early excavation of the hamate on day 6 . MafB staining is predominantly nuclear; however, cytoplasmic staining (less intense than the nuclear staining) also is seen, as highlighted in D (arrowheads). G: MafB-expressing cells with ramified macrophage-like morphology are observed (arrowheads). J: A multinucleated osteoclast is visible (arrowhead), showing both nuclear and cytoplasmic staining. P-S: Isotype control staining shows low-level nonspecific cartilage matrix staining, but no cellular staining. Orientation of images is with distal-most tissue in top left corner. Original magnification: $\times 40$. Scale bars: $20 \mu \mathrm{m}(\mathbf{A}-\mathbf{0})$. D, day; Iso, isotype; W, week.
} 


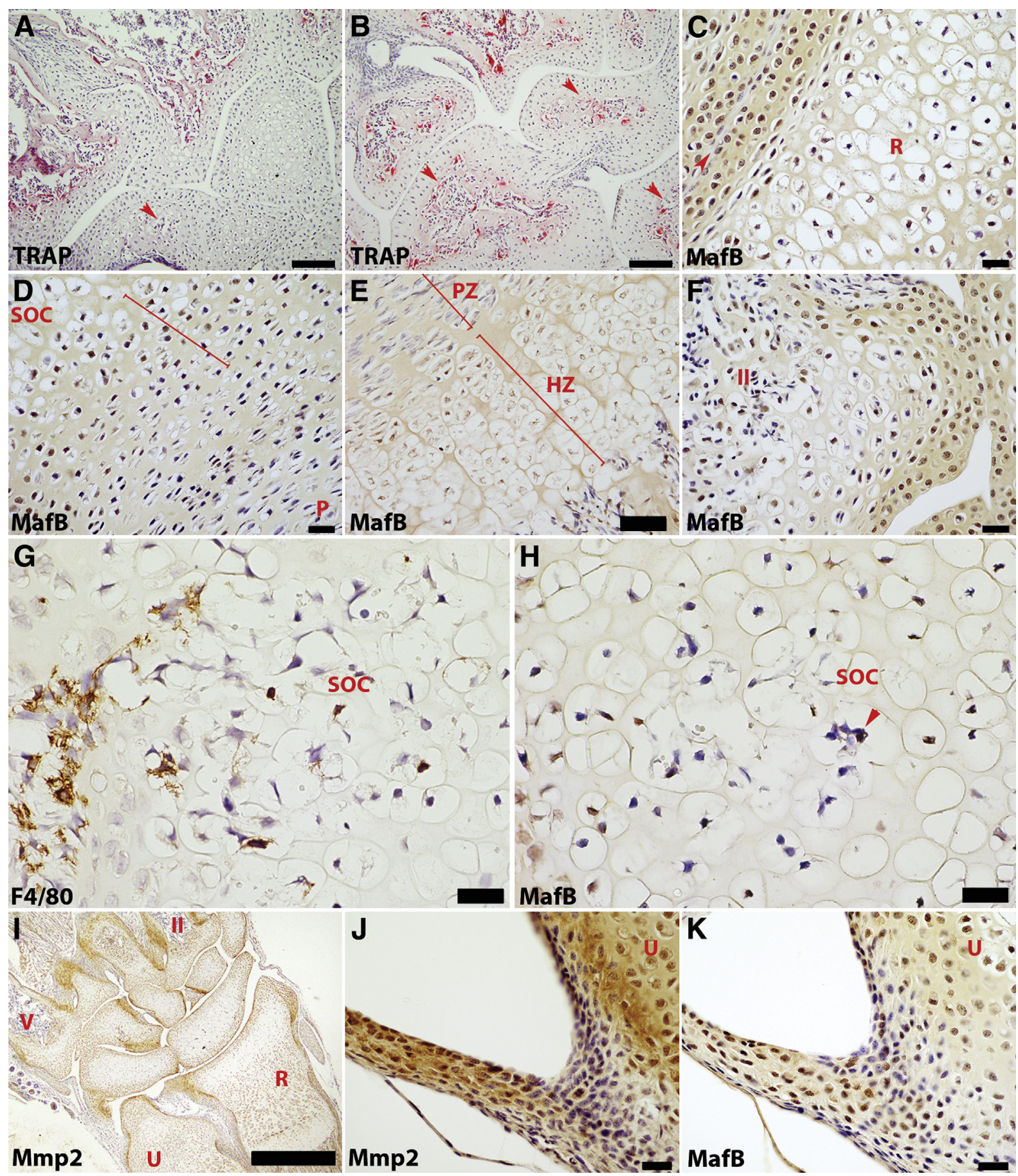

Figure 5 Histologic characteristics of extracarpal wrist tissue. A: On day 8, a small tartrate-resistant acid phosphatase (TRAP) ${ }^{+}$region (pink stain) was visible within the hamate excavation site (arrowhead); there was much more intense staining of the proximal metacarpals. B: 0n day 12, all visible carpals were TRAP ${ }^{+}$ (arrowheads). C: V-maf musculoaponeurotic fibrosarcoma oncogene ortholog B (MafB) staining of articular cartilage and secondary ossification centers (SOCs) of the distal radius on day 6 showing near-universal staining of articular chondrocytes, and more variable staining within the radial SOC. Arrowhead indicates the articular surface between the navicular lunate and radius. D: MafB staining of prephyseal chondrocytes (red line) on day 6 , showing a similar pattern of staining to carpal primary ossification centers (POCs). E: MafB staining of physeal hypertrophic chondrocytes on day 8 showed MafB ${ }^{-}$cells within the prehypertrophic zone $(\mathrm{PZ})$ and $\mathrm{MafB}^{+}$cells within the hypertrophic zone (HZ). F: MafB staining of the proximal second metacarpal on day 6 , showing a similar pattern of staining to carpal POCs. G: $\mathrm{F} 4 / 80$ staining of radial SOCs on day 8 showing a loose line of $\mathrm{F} 4 / 80^{+}$macrophages connecting the medial edge of the radius with the early SOC, and scattered $\mathrm{F}_{4} / 80^{+}$cells within the SOC. H: Serial MafB staining, with arrowhead indicating a $\mathrm{MafB}^{+}$cell with macrophage-like morphology. I: Matrix metalloproteinase (Mmp)-2 staining on day 6, showing marked entheseal staining. J: Mmp-2 staining of the distal ulna and adjacent lateral enthesis on day 6 , showing intense cellular and matrix staining. K: Similar distribution of MafB nuclear staining. Thus, TRAP ${ }^{+}$cells are present only after excavation has commenced, whereas MafB is present earlier within subarticular cartilage, regardless of whether this is carpal or extracarpal, and in nonbone noncartilage tissues. Scale bars: $100 \mu \mathrm{m}$ $(\mathbf{A}$ and $\mathbf{B}) ; 20 \mu \mathrm{m}(\mathbf{C}, \mathbf{D}, \mathbf{F}-\mathbf{H}, \mathbf{J}$, and $\mathbf{K}) ; 50 \mu \mathrm{m}(\mathbf{E}) ; 500 \mu \mathrm{m}(\mathbf{I})$. Original magnification: $\times 20(\mathbf{A}) ; \times 60(\mathbf{C}, \mathbf{D}, \mathbf{F}, \mathbf{G}$, and J); $\times 40(\mathbf{E}) ; \times 4(\mathbf{I})$. II, second metacarpal; $P$, physis; $R$, distal radius; $U$, distal ulna; $V$, fifth metacarpal. 
confirmed. Mmp-14-deficient mice also show near-normal physis development with only transient abnormalities of the POC, ${ }^{27}$ but have delayed and abnormal postnatal SOC development, abnormal entheseal remodeling, and progressive entheseal fibrosis, with an associated increase in osteoclastic bone resorption. ${ }^{44}$

Overall, this study has identified that, in mice, an endochondral ossification mechanism (subarticular endochondral ossification) distinct from archetypical physeal endochondral ossification, is responsible for the formation of the carpal bones as well as the distal ulna and radius, and the second to fifth proximal metacarpals. Subarticular endochondral ossification progressed via complete mineralization of a cartilaginous template of the mature bone structure with delayed vascular invasion and osteoclast-mediated remodeling relative to physeal growth plate temporal events. MafB, Mmp-2, and Mmp-14 were expressed within chondrocytes from the earliest stages of subarticular endochondral ossification. Because the skeletal abnormalities of CTODs predominantly affect wrist bones that develop via subarticular endochondral ossification, our accumulated data provide plausible explanations for the site-specific distribution of bone abnormalities and overlapping phenotypes of the three prototypical CTODs. These observations provide a valuable roadmap for future investigation of the underlying pathologic features of these syndromes using appropriate animal models. Our findings raise the possibility that the underlying abnormality of CTODs may be impaired bone modeling rather than an osteolytic pathology, although secondary dysregulated or compensatory osteoclastic activity cannot be ruled out. If correct, this would shift our understanding of these conditions from osteolytic disorders to disorders of dysfunctional skeletal formation, with consequent implications for treatment and nomenclature.

\section{Acknowledgments}

We thank the Translational Research Institute Histology Core and Preclinical Imaging Facilities and the Queensland Institute for Medical Research Berghofer Histotechnology Facility for technical support, Prof. Matthew Brown for his advice and support throughout the study, and Dr Julien Legrand and The University of Queensland Biological Research Facility for the supply of mouse neonates.

S.L. conceptualized and designed the study, obtained ethics clearance, performed the bulk of the experiments, analyzed data, and drafted and edited the manuscript; H.-W.T. assisted with experiments and approved the final manuscript; F.L. and M.A.W. designed and performed some experiments and approved the final manuscript; E.L.D. conceptualized and designed the study and edited the manuscript; and A.R.P. conceptualized and designed the study, obtained ethics clearance, analyzed data, and edited the manuscript.

\section{Supplemental Data}

Supplemental material for this article can be found at http://dx.doi.org/10.1016/j.ajpath.2017.05.007.

\section{References}

1. Warman ML, Cormier-Daire V, Hall C, Krakow D, Lachman R, LeMerrer M, Mortier G, Mundlos S, Nishimura G, Rimoin DL, Robertson S, Savarirayan R, Sillence D, Spranger J, Unger S, Zabel B, Superti-Furga A: Nosology and classification of genetic skeletal disorders: 2010 revision. Am J Med Genet A 2011, 155A:943-968

2. Zankl A, Duncan EL, Leo PJ, Clark GR, Glazov EA, Addor MC, Herlin T, Kim CA, Leheup BP, McGill J, McTaggart S, Mittas S, Mitchell AL, Mortier GR, Robertson SP, Schroeder M, Terhal P, Brown MA: Multicentric carpotarsal osteolysis is caused by mutations clustering in the amino-terminal transcriptional activation domain of MAFB. Am J Hum Genet 2012, 90:494-501

3. Martignetti JA, Aqeel AA, Sewairi WA, Boumah CE, Kambouris M, Mayouf SA, Sheth KV, Eid WA, Dowling O, Harris J, Glucksman MJ, Bahabri S, Meyer BF, Desnick RJ: Mutation of the matrix metalloproteinase 2 gene (MMP2) causes a multicentric osteolysis and arthritis syndrome. Nat Genet 2001, 28:261-265

4. Evans BR, Mosig RA, Lobl M, Martignetti CR, Camacho C, GrumTokars V, Glucksman MJ, Martignetti JA: Mutation of membrane type-1 metalloproteinase, MT1-MMP, causes the multicentric osteolysis and arthritis disease Winchester syndrome. Am J Hum Genet 2012, 91:572-576

5. Al Aqeel A, Al Sewairi W, Edress B, Gorlin RJ, Desnick RJ, Martignetti JA: Inherited multicentric osteolysis with arthritis: a variant resembling Torg syndrome in a Saudi family. Am J Med Genet 2000, 93:11-18

6. Winchester P, Grossman H, Lim WN, Danes BS: A new acid mucopolysaccharidosis with skeletal deformities simulating rheumatoid arthritis. Am J Roentgenol Radium Ther Nucl Med 1969, 106: $121-128$

7. Faber MR, Verlaak R, Fiselier TJ, Hamel BC, Franssen MJ, Gerrits GP: Inherited multicentric osteolysis with carpal-tarsal localisation mimicking juvenile idiopathic arthritis. Eur J Pediatr 2004, 163: 612-618

8. Carnevale A, Canun S, Mendoza L, del Castillo V: Idiopathic multicentric osteolysis with facial anomalies and nephropathy. Am J Med Genet 1987, 26:877-886

9. Mehawej C, Courcet JB, Baujat G, Mouy R, Gerard M, Landru I, Gosselin M, Koehrer P, Mousson C, Breton S, Quartier P, Le Merrer M, Faivre L, Cormier-Daire V: The identification of MAFB mutations in eight patients with multicentric carpo-tarsal osteolysis supports genetic homogeneity but clinical variability. Am J Med Genet A 2013, 161A:3023-3029

10. Rouzier C, Vanatka R, Bannwarth S, Philip N, Coussement A, PaquisFlucklinger V, Lambert JC: A novel homozygous MMP2 mutation in a family with Winchester syndrome. Clin Genet 2006, 69:271-276

11. Castberg FC, Kjaergaard S, Mosig RA, Lobl M, Martignetti C, Martignetti JA, Myrup C, Zak M: Multicentric osteolysis with nodulosis and arthropathy (MONA) with cardiac malformation, mimicking polyarticular juvenile idiopathic arthritis: case report and literature review. Eur J Pediatr 2013, 172:1657-1663

12. Zankl A, Bonafe L, Calcaterra V, Di Rocco M, Superti-Furga A: Winchester syndrome caused by a homozygous mutation affecting the active site of matrix metalloproteinase 2. Clin Genet 2005, 67: 261-266

13. Wenkert D, Mumm S, Wiegand SM, McAlister WH, Whyte MP: Absence of MMP2 mutation in idiopathic multicentric osteolysis with nephropathy. Clin Orthop Relat Res 2007, 462:80-86 
14. Phadke SR, Ramirez M, Difeo A, Martignetti JA, Girisha KM: Torg-Winchester syndrome: lack of efficacy of pamidronate therapy. Clin Dysmorphol 2007, 16:95-100

15. Tuysuz B, Mosig R, Altun G, Sancak S, Glucksman MJ, Martignetti JA: A novel matrix metalloproteinase 2 (MMP2) terminal hemopexin domain mutation in a family with multicentric osteolysis with nodulosis and arthritis with cardiac defects. Eur J Hum Genet 2009, 17:565-572

16. Goldfarb CA, Steffen JA, Whyte MP: Idiopathic multicentric osteolysis: upper extremity manifestations and surgical considerations during childhood. J Hand Surg Am 2012, 37:1677-1683

17. Erickson CM, Hirschberger M, Stickler GB: Carpal-tarsal osteolysis. J Pediatr 1978, 93:779-782

18. Mumm S, Huskey M, Duan S, Wenkert D, Madson KL, Gottesman GS, Nenninger AR, Laxer RM, McAlister WH, Whyte MP: Multicentric carpotarsal osteolysis syndrome is caused by only a few domain-specific mutations in MAFB, a negative regulator of RANKLinduced osteoclastogenesis. Am J Med Genet A 2014, 164A: 2287-2293

19. Zankl A, Pachman L, Poznanski A, Bonafe L, Wang F, Shusterman Y, Fishman DA, Superti-Furga A: Torg syndrome is caused by inactivating mutations in MMP2 and is allelic to NAO and Winchester syndrome. J Bone Miner Res 2007, 22:329-333

20. Galasso O, Familiari F, De Gori M, Gasparini G: Recent findings on the role of gelatinases (matrix metalloproteinase-2 and -9) in osteoarthritis. Adv Orthop 2012, 2012:834208

21. Kim K, Kim JH, Lee J, Jin HM, Kook H, Kim KK, Lee SY, Kim N: MafB negatively regulates RANKL-mediated osteoclast differentiation. Blood 2007, 109:3253-3259

22. Menendez-Gutierrez MP, Roszer T, Fuentes L, Nunez V, Escolano A, Redondo JM, De Clerck N, Metzger D, Valledor AF, Ricote M: Retinoid X receptors orchestrate osteoclast differentiation and postnatal bone remodeling. J Clin Invest 2015, 125:809-823

23. Sakai M, Imaki J, Yoshida K, Ogata A, Matsushima-Hibaya Y, Kuboki Y, Nishizawa M, Nishi S: Rat maf related genes: specific expression in chondrocytes, lens and spinal cord. Oncogene 1997, 14: $745-750$

24. Zhang Y, Ross AC: Retinoic acid and the transcription factor MafB act together and differentially to regulate aggrecan and matrix metalloproteinase gene expression in neonatal chondrocytes. J Cell Biochem 2013, 114:471-479

25. Edwards JC, Wilkinson LS, Soothill P, Hembry RM, Murphy G, Reynolds JJ: Matrix metalloproteinases in the formation of human synovial joint cavities. J Anat 1996, 188:355-360

26. Duerr S, Stremme S, Soeder S, Bau B, Aigner T: MMP-2/gelatinase A is a gene product of human adult articular chondrocytes and is increased in osteoarthritic cartilage. Clin Exp Rheumatol 2004, 22: 603-608

27. Mosig RA, Dowling O, DiFeo A, Ramirez MC, Parker IC, Abe E, Diouri J, Aqeel AA, Wylie JD, Oblander SA, Madri J, Bianco P, Apte SS, Zaidi M, Doty SB, Majeska RJ, Schaffler MB, Martignetti JA: Loss of MMP-2 disrupts skeletal and craniofacial development and results in decreased bone mineralization, joint erosion and defects in osteoblast and osteoclast growth. Hum Mol Genet 2007, 16:1113-1123

28. Standring S: Pectoral girdle and upper limb. Gray's Anatomy, ed 40 Edited by Johnson D. Spain: Churchill Livingstone Elsevier, 2008. pp. $45-52$
29. Berner A, Reichert JC, Woodruff MA, Saifzadeh S, Morris AJ, Epari DR, Nerlich M, Schuetz MA, Hutmacher DW: Autologous vs. allogenic mesenchymal progenitor cells for the reconstruction of critical sized segmental tibial bone defects in aged sheep. Acta Biomater 2013, 9:7874-7884

30. Chang MK, Raggatt LJ, Alexander KA, Kuliwaba JS, Fazzalari NL, Schroder K, Maylin ER, Ripoll VM, Hume DA, Pettit AR: Osteal tissue macrophages are intercalated throughout human and mouse bone lining tissues and regulate osteoblast function in vitro and in vivo. J Immunol 2008, 181:1232-1244

31. Bab I, Hajbi-Yonissi C, Gabet Y, Muller R: Forearm (ulna, radius, and elbow joint). Manus. Micro-Tomographic Atlas of the Mouse Skeleton. Jerusalem: Springer Science+Business Media, 2007. pp. $131-149$

32. Glowacki J, Rey C, Glimcher MJ, Cox KA, Lian J: A role for osteocalcin in osteoclast differentiation. J Cell Biochem 1991, 45: 292-302

33. Gilsanz V, Ratib O: Hand Bone Age: A Digital Atlas of Skeletal Maturity. Berlin: Springer-Verlag Berlin, 2005. pp. 35-63

34. Shurtleff DB, Sparkes RS, Clawson DK, Guntheroth WG, Mottet NK: Hereditary osteolysis with hypertension and nephropathy. JAMA 1964, 188:363-368

35. Torg JS, DiGeorge AM, Kirkpatrick JA Jr, Trujillo MM: Hereditary multicentric osteolysis with recessive transmission: a new syndrome. J Pediatr 1969, 75:243-252

36. Jerome C, Hoch B: Skeletal System. Edited by Treuting PM, Dintzis S, Liggitt D, Frevert CW. Amsterdam: Elsevier, 2012

37. Ogden JA, Ganey TM, Light TR, Belsole RJ, Greene TL: Ossification and pseudoepiphysis formation in the "nonepiphyseal" end of bones of the hands and feet. Skeletal Radiol 1994, 23:3-13

38. Reno PL, McBurney DL, Lovejoy CO, Horton WE Jr: Ossification of the mouse metatarsal: differentiation and proliferation in the presence/absence of a defined growth plate. Anat Rec A Discov Mol Cell Evol Biol 2006, 288:104-118

39. Hita-Contreras F, Martinez-Amat A, Ortiz R, Caba O, Alvarez P, Prados JC, Lomas-Vega R, Aranega A, Sanchez-Montesinos I, Merida-Velasco JA: Development and morphogenesis of human wrist joint during embryonic and early fetal period. J Anat 2012, 220:580-590

40. Rivas R, Shapiro F: Structural stages in the development of the long bones and epiphyses: a study in the New Zealand white rabbit. J Bone Joint Surg Am 2002, 84-A:85-100

41. Ganey TM, Love SM, Ogden JA: Development of vascularization in the chondroepiphysis of the rabbit. J Orthop Res 1992, 10:496-510

42. Lagier R, Rutishauser E: Osteoarticular changes in a case of essential osteolysis; an anatomical and radiological study. J Bone Joint Surg Br 1965, 47:339-353

43. Pai GS, Macpherson RI: Idiopathic multicentric osteolysis: report of two new cases and a review of the literature. Am J Med Genet 1988, 29:929-936

44. Holmbeck K, Bianco P, Caterina J, Yamada S, Kromer M, Kuznetsov SA, Mankani M, Robey PG, Poole AR, Pidoux I, Ward JM, Birkedal-Hansen H: MT1-MMP-deficient mice develop dwarfism, osteopenia, arthritis, and connective tissue disease due to inadequate collagen turnover. Cell 1999, 99:81-92

45. Inoue K, Mikuni-Takagaki Y, Oikawa K, Itoh T, Inada M, Noguchi T, Park JS, Onodera T, Krane SM, Noda M, Itohara S: A crucial role for matrix metalloproteinase 2 in osteocytic canalicular formation and bone metabolism. J Biol Chem 2006, 281:33814-33824 